\title{
Lista de Revisores / List of reviewers 2015
}

Acuña, Marcelo (Argentina)

Akita, Keiichi (Japón)

Amin, Sameth (Egipto)

Apaydin, Nihal (Turquía)

Armand Ugón, Gustavo (Uruguay)

Arraez-Aybar, Luis A. (España)

Aziz, Josef (Libia)

Barros, Mirna D (Brasil)

Beltramino, Raúl (Argentina)

Bucarey, Sandra (Chile)

Campero, A. Álvaro (Argentina)

Chitra, Ramasamy (India)

Correa, Leandro J. (Argentina)

Criado del Río, Luis E. (Argentina)

De Caro, Raffaele (Italia)

Eid, Nabil (Japón)

Fogg, Quentin (Escocia)

Haviarová, Zora (Eslovaquia)

Hayes, Susan (Australia)

Ikuta, Carla R. S. (Brasil)

Jacks, Tamunotubo W. (Nigeria)

Komarnitki, Iulian (Polonia)

Krishan, Kewal (India)

Mansur, Dil I. (Nepal)
Martino, Elia del C. (Argentina)

Matsutani, Shinji (Japón)

Mechirová, Eva (Eslovaquia)

Minhas, Liagat A. (Arabia Saudita)

Mompeó Corredera, Blanca R. (España)

Murlimanju, Bukkambudhi V. (India)

Musa, Ahmed (Rumania)

Mustafa, Ayman G. (Jordania)

Nor, Faridah M. (Malasia)

Paraskevas, George K. (Grecia)

Piagkou, Mara (Grecia)

Sampietro, Agustín (Argentina)

Sañudo, José R. (España)

Spitale, Luis S. (Argentina)

Srivastava, Sushil K. (India)

Stigliano, Javier (Argentina)

Tohno, Yoshiyuki (Tailandia)

Toledo Neto, Joao L. (Brasil)

Totlis, Trifon (Grecia)

Urrets Zavalía, Enrique (Argentina)

Vatsalaswamy, Puranam (India)

Vlajkovic, Slobodan (Serbia)

Ward, Roberta J. (Reino Unido)

Wessels, Quenton (Reino Unido) 